\section{Commentary: Survival following extracorporeal membrane oxygenation support, a sobering view}

\author{
Michel Carrier, MD, MBA
}

Extracorporeal membrane oxygenation (ECMO) mechanical support is applied nowadays to a variety of patients with numerous clinical conditions and various diagnoses. The common denominator for all patients is refractory cardiogenic shock.

In the current issue of the Journal, Sertic and colleagues ${ }^{1}$ reviewed a cohort of 92 patients who survived the first 24 hours following successful weaning of ECMO support. Patient survival reached $64 \%$ at hospital discharge and $55 \%$ at 1 year in this cohort. The investigators sought to evaluate the risk factors associated with poor outcomes in this group of patients who survived at least 24 hours following the weaning protocol. This group represents one of the best clinical scenarios in terms of cardiac recovery and can be considered to have a successful outcome of ECMO support. Yet, only 1 in 2 patients will be alive 1 year after the episode of care? Moreover, the 1-year survival of patients who were successfully discharged from the hospital after a median stay of 24 days was $84 \%$ ! We also have to state that in total 289 patients died on ECMO and 28 patients died within 24 hours of weaning from ECMO and were excluded from the present analysis. Another group of 43 patients failed ECMO weaning and underwent heart-replacement therapy with left ventricular assist device (VAD) or heart transplantation.

Diabetes, previous myocardial infarction, prolonged ECMO support, hypoxemia at ECMO weaning, left ventricular dysfunction, and mitral regurgitation were the risk factors for in-hospital mortality after weaning. Of interest,

\footnotetext{
From the Division of Cardiac Surgery, Department of Surgery, University of Montreal and Montreal Heart Institute, Montréal, Québec, Canada.

Disclosures: Author has nothing to disclose with regard to commercial support.

Received for publication Nov 27, 2019; revisions received Nov 27, 2019; accepted for publication Nov 29, 2019; available ahead of print Dec 14, 2019.

Address for reprints: Michel Carrier, MD, MBA, 5000 rue Bélanger Est, Montréal, Québec, Canada, H1T 1C8 (E-mail: michel.carrier@icm-mhi.org).

J Thorac Cardiovasc Surg 2021;161:679-80

$0022-5223 / \$ 36.00$

Copyright (C) 2019 by The American Association for Thoracic Surgery

https://doi.org/10.1016/j.jtcvs.2019.11.115
}

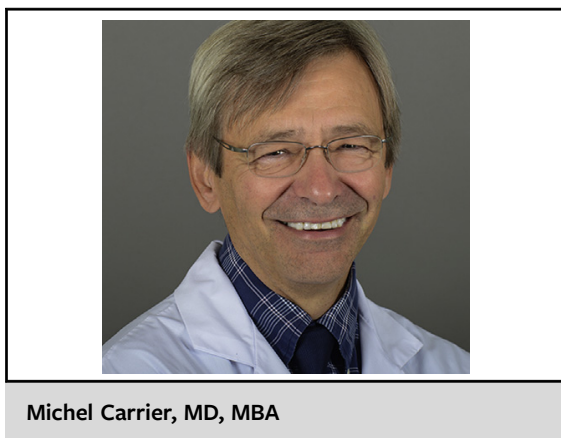

CENTRAL MESSAGE

The present study shows that successful weaning from ECMO support is by no means a guarantee of survival at 1 year. Much more work needs to be done to ensure an acceptable rate of survival.

$74 \%$ of patients who underwent heart-replacement therapy with VADs and heart transplantation were alive at 1 year.

Results from the present analyses are interesting but stress several points of failure. First, we desperately need more stringent patient indications to implement mechanical support because $73 \%(349 / 480)$ of the patients died inhospital during or immediately after ECMO support. Second, we also desperately need a better approach to ensure myocardial recovery during and after mechanical support. Left ventricular unloading may be an answer, but it needs serious documentation., ${ }^{2,3}$ Third, we need to implement better protocols of interventions and follow-up because 35\% (32/92) of patients successfully weaned from ECMO died in-hospital and a few more end up dying after hospital discharge with an overall patient survival at 1 year reaching only $55 \%$.

The present study brings a sobering view to the current results of our initial strategy for mechanical support of the failing heart. Large gaps in knowledge remain, especially in approaches to myocardial recovery. Moreover, successful weaning from ECMO support is by no means a guarantee of survival at 1 year. What is the role of heart-replacement therapy with left VADs, heart transplantation, and the total artificial heart in these patients? With a $74 \%$ survival at 1 year, these should be serious options.

The authors rightly concluded that the decrease in patient survival following successful ECMO weaning is an 
important signal that requires analysis in an attempt to improve patient outcomes. So much work needs to be done!

\section{References}

1. Sertic F, Chavez L, Diagne D, Richards T, Wald J, Acker M, et al. Predictors of in-hospital mortality and midterm outcomes of patients successfully weaned from venoarterial extracorporeal membrane oxygenation. J Thorac Cardiovasc Surg. 2021;161:666-78.e3.

2. Russo JJ, Aleksova N, Pitcher I, Couture E, Parlow S, Faraz M, et al. Left ventricular unloading during extracorporeal membrane oxygenation in patients with cardiogenic shock. J Am Coll Cardiol. 2019;73:654-62.

3. Pappalardo F, Schulte C, Pieri M, Schrage B, Contri R, Soeffker G, et al. Concomitant implantation of Impella(R) on top of veno-arterial extracorporeal membrane oxygenation may improve survival of patients with cardiogenic shock. Eur J Heart Fail. 2017;19:404-12. 